\title{
Some Limiting Cases of the G-Transformation*
}

\author{
By H. L. Gray and W. R. Schucany
}

\begin{abstract}
In this paper some new nonlinear transformations are introduced. They arise from considering the limit of the $G$-transformation as a particular parameter approaches its limiting value. The primary purpose of these transformations is to increase the rate of convergence of an improper integral. However, by introduction of an iteration method it is shown that they may also be used to produce approximating functions for the tail of an improper integral. Several examples are included.
\end{abstract}

I. Introduction. In [6], [8], [11] H. L. Gray and T. A. Atchison have introduced some transformations which are of some value in evaluating improper integrals. In general, these transformations are a function of an unknown parameter $k$. Moreover, in [11] it was demonstrated that for some cases these transformations are increasingly effective as $k$ approaches a limiting value. This latter observation has, in fact, been used by Gray and Schucany [10] and Gray, McWilliams and Thompson [12] to produce approximating functions for Mill's ratio, and the Chi-square integral. In both of these latter papers only a limiting case of the so-called $G$-transformation was used. In this paper the limiting transformations of both the $G$ and $B$ transformations of [11] are considered in somewhat more detail than previously studied. Illustrative examples are included. For clarity we now give the following definitions.

Definition 1. Let $f$ be a real valued function of a real variable $x$ such that $f$ is continuous for $a \leqq x<\infty$ and $F(t)=\int_{a}^{t} f(x) d x$ converges as $t \rightarrow \infty$. Further, let

$$
R_{1}(t ; k)=\frac{f(t+k)}{f(t)}
$$

and

$$
R_{2}(t ; k)=\frac{k f(k t)}{f(t)}
$$

if $f(t) \neq 0$. If $f\left(t_{0}\right)=0$ and $\lim _{t \rightarrow t_{0}} R_{1}(t ; k)$ and $\lim _{t \rightarrow t_{0}} R_{2}(t ; k)$ exist, we define $R_{1}\left(t_{0} ; k\right)$ and $R_{2}\left(t_{0} ; k\right)$ to be those limits. Finally, if $R_{1}(t ; k)$ and $R_{2}(t ; k)$ are defined and different from 1 , then we define the $G$ and $B$ transformations by

$$
G[F ; t ; k]=\frac{F(t+k)-R_{1}(t ; k) F(t)}{1-R_{1}(t ; k)}
$$

and

$$
B[F ; t ; k]=\frac{F(k t)-R_{2}(t ; k) F(t)}{1-R_{2}(t ; k)} .
$$

Received December 26, 1968.

* This research was partially supported by ONR Contract N00014-68-A-0515. 
Note that the condition $R_{1}(t ; k)$ and $R_{2}(t ; k) \neq 1$ requires $k \neq 0$ in (1.3) and $k \neq 1$ in (1.4).

As mentioned above, in [11] it was shown that in some cases (1.3) and (1.4) give increasingly better approximations for $F(\infty)$ as $k \rightarrow 0$ and $k \rightarrow 1$ respectively. To exploit this idea we shall examine these limiting processes in the following theorems.

\section{A New Class of Transformations.}

Theorem 1. If $f$ is differentiable on $(a, \infty)$ and $F$ is the function described in Definition 1, then when the $\lim _{k \rightarrow 1} B[F ; t, k]$ exists we have

$$
\lim _{k \rightarrow 1} B[F ; t, k]=F(t)-\frac{t f^{2}(t)}{t f^{\prime}(t)+f(t)}, \text { when } t f^{\prime}(t)+f(t) \neq 0 .
$$

[In the above and in that which follows the prime will always denote differentiation with respect to the argument $t$.]

Proof.

$$
\begin{aligned}
\lim _{k \rightarrow 1} B[F ; t, k] & =\lim _{k \rightarrow 1} \frac{F(k t)-R_{2}(t ; k) F(t)}{1-R_{2}(t ; k)} \\
& =\lim _{k \rightarrow 1} \frac{f(t) F(k t)-k f(k t) F(t)}{f(t)-k f(k t)} \\
& =\lim _{k \rightarrow 1} \frac{F(t)[k f(k t)-f(t)]-f(t)[F(k t)-F(t)]}{k f(k t)-f(t)} \\
& =\lim _{k \rightarrow 1}\left\{F(t)-\frac{f(t)(F(k t)-F(t)) /(k-1)}{(k f(k t)-f(t)) /(k-1)}\right\} .
\end{aligned}
$$

Next, let $k=1+\Delta k$ and the above limit becomes

$$
F(t)-\lim _{\Delta k \rightarrow 0} \frac{f(t)(F(t+t \Delta k)-F(t)) / \Delta k}{(f(t+t \Delta k)-f(t)) / \Delta k+f(t+t \Delta k)} .
$$

Hence, letting $\Delta t=t \Delta k$ we have

$$
\begin{aligned}
\lim _{k \rightarrow 1} B[F ; t, k] & =F(t)-\lim _{\Delta t \rightarrow 0} \frac{t f(t)(F(t+\Delta t)-F(t)) / \Delta t}{t(f(t+\Delta t)-f(t)) / \Delta t+f(t+\Delta t)} \\
& =F(t)-\frac{t f^{2}(t)}{t f^{\prime}(t)+f(t)} .
\end{aligned}
$$

Theorem 2. If $f$ and $F$ are the functions described in Theorem 1, then

$$
\lim _{k \rightarrow 0} G[F ; t, k]=F(t)-\frac{f^{2}(t)}{f^{\prime}(t)}, \text { when } f^{\prime}(t) \neq 0 .
$$

In the above we are of course assuming the existence of

$$
\lim _{k \rightarrow 0} G[F ; t, k] \text {. }
$$

Proof. The proof is somewhat similar to that of Theorem 1 and hence will not be given. 
Clearly Theorems 1 and 2 lead us to two distinct limiting transformations. However, due to the similarities between these two transformations, it is possible to combine both of them under one definition as follows:

Definition 2. For the functions $f$ and $F$ previously discussed we define the $C_{p}$ transformation by

$$
\begin{aligned}
C_{p}[F(t)] & =F(t)-\frac{t f^{2}(t)}{t f^{\prime}(t)+p f(t)} \\
& =F(t)-\frac{t^{p} f^{2}(t)}{\left[t^{p} f(t)\right]^{\prime}}, \quad 0 \leqq p \leqq 1 .
\end{aligned}
$$

Obviously when $p=1$ and $p=0,(2.10)$ becomes (2.1) and (2.9) respectively. The full significance of the parameter $p$ in (2.10) has not been established. However, (2.10) has the immediate advantage of unifying (2.1) and (2.9) and hence eliminating the necessity of studying them separately.

For clarity we include one additional definition.

Definition 3. If $A(t)$ and $B(t)$ are two sequences of real numbers such that $\lim _{t \rightarrow \infty} A(t)=A \neq \pm \infty$ and $\lim _{t \rightarrow \infty} B(t)=B \neq \pm \infty$, then we say $A(t)$ converges uniformly better than $B(t)$ on $(a, b)$ if and only if

$$
|A-A(t)|<|B-B(t)|
$$

for every $t \in(a, b)$.

Further, if

$$
\lim _{t \rightarrow \infty}\left|\frac{A-A(t)}{B-B(t)}\right|=0
$$

then we say $A(t)$ converges more rapidly than $B(t)$.

Theorem 3. If $A(t)$ converges more rapidly than $B(t)$, then there exists an $a_{0}$ such that $A(t)$ converges uniformly better than $B(t)$ on $\left(a_{0}, \infty\right)$.

Proof. The result is obvious.

Theorem 4. If $\lim _{t \rightarrow \infty}|(A-A(t)) /(B-B(t))|$ exists and is not equal to one, then a necessary and sufficient condition that there exist an $a_{0}$ such that $A(t)$ converges uniformly better than $B(t)$ on $\left(a_{0}, \infty\right)$ is that

$$
\lim _{t \rightarrow \infty}\left|\frac{A-A(t)}{B-B(t)}\right|<1 .
$$

Proof. The proof is quite simple and hence will not be given.

Theorem 5. If

$$
\lim _{t \rightarrow \infty} \frac{F(\infty)-C_{p}[F(t)]}{F(\infty)-F(t)} \neq 1
$$

but does exist, then a necessary and sufficient condition that there exists an $a_{0}$ such that $C_{p}[F(t)]$ converges uniformly better than $F(t)$ to $F(\infty)$ is

$$
0<\lim _{t \rightarrow \infty} \frac{t f^{2}(t)}{\left[t f^{\prime}(t)+p f(t)\right] E(t)}<2
$$


or

$$
0<\lim _{t \rightarrow \infty} \frac{t^{p} f(t)}{\left[t^{p} f(t)\right]^{\prime}} \frac{f(t)}{E(t)}<2
$$

where $E(t)=F(t)-F(\infty)$.

Proof. By the previous theorem a necessary and sufficient condition that $C_{p}[F(t)]$ convergence uniformly better than $F(t)$ to $F(\infty)$ on some interval $\left(a_{0}, \infty\right)$ is that

$$
-1<\lim _{t \rightarrow \infty} \frac{F(\infty)-\left\{F(t)-t f^{2}(t) /\left(t f^{\prime}(t)+p f(t)\right)\right\}}{F(\infty)-F(t)}<1 .
$$

The latter is true, however, if and only if (2.15) holds and the theorem follows. It should be pointed out that (2.15) is useful although at first glance it appears to require $F(\infty)$ to establish whether or not it is satisfied. This, of course, may not be the case as will be demonstrated in a later example.

THEOREM 6. A necessary and sufficient condition that $C_{p}[F(t)]$ converge more rapidly than $F(t)$ to $F(\infty)$ is that

$$
\lim _{t \rightarrow \infty} \frac{t f^{2}(t)}{\left[t f^{\prime}(t)+p f(t)\right] E(t)}=1
$$

where $E(t)=F(t)-F(\infty)$.

Proof. We note that

$$
\frac{F(\infty)-C_{p}[F(t)]}{F(\infty)-F(t)}=1-\frac{t f^{2}(t)}{\left[t f^{\prime}(t)+p f(t)\right] E(t)}
$$

and the result follows.

Theorem 7. If $\lim _{t \rightarrow \infty} f(t)$ and $\lim _{t \rightarrow \infty} f(t) / f^{\prime}(t)$ exist (finite) and the latter is not zero, then $C_{p}[F(t)]$ converges more rapidly than $F(t)$ to $F(\infty)$.

Proof.

$$
\lim _{t \rightarrow \infty} \frac{t f^{2}(t)}{\left[t f^{\prime}(t)+p f(t)\right] E(t)}=\lim _{t \rightarrow \infty} \frac{f(t) / f^{\prime}(t)}{1+(p / t) f(t) / f^{\prime}(t)} \cdot \frac{f(t)}{E(t)} .
$$

Now since $F(\infty)$ exists and $\lim _{t \rightarrow \infty} f(t)$ exists, then $\lim _{t \rightarrow \infty} f(t)=0$. Thus since $\lim _{t \rightarrow \infty} f(t) / f^{\prime}(t)$ exists, we have

$$
\lim _{t \rightarrow \infty} \frac{f(t) / f^{\prime}(t)}{1+(p / t) f(t) / f^{\prime}(t)}=\lim _{t \rightarrow \infty} \frac{f(t)}{f^{\prime}(t)}=\lim _{t \rightarrow \infty} \frac{E(t)}{f(t)}
$$

by L'Hospital's rule. Now since this limit is not zero, the result follows immediately from $(2.20)$.

TheOREM 8. If $f(t)$ has a countable number of zeros, $\lim _{t \rightarrow \infty} t f(t)$ exists and the $\lim _{t \rightarrow \infty} f(t) /[t f(t)]^{\prime}$ exists and is different from zero, then $C_{p}[F(t)]$ converges uniformly better to $F(\infty)$ than $F(t)$ on some interval $\left(a_{0}, \infty\right)$ for every $p$. Moreover, if $p=1$, then $C_{p}[F(t)]$ converges more rapidly to $F(\infty)$ than $F(t)$. 
Proof.

$$
\begin{aligned}
\lim _{t \rightarrow \infty} \frac{t f^{2}(t)}{\left[t f^{\prime}(t)+p f(t)\right] E(t)} & =\lim _{t \rightarrow \infty} \frac{f(t)}{t f^{\prime}(t)+p f(t)} \cdot \frac{t f(t)}{E(t)} \\
& =\lim _{t \rightarrow \infty} \frac{[t f(t)]^{\prime}}{[t f(t)]^{\prime}+(p-1) f(t)} \cdot \frac{f(t)}{[t f(t)]^{\prime}} \frac{t f(t)}{E(t)} \\
& =\lim _{t \rightarrow \infty} \frac{[t f(t)]^{\prime} / f(t)}{[t f(t)]^{\prime} / f(t)+(p-1)} \cdot \frac{f(t)}{[t f(t)]^{\prime}} \cdot \frac{t f(t)}{E(t)} .
\end{aligned}
$$

Now the limit of each of the factors in (2.23) exist by our assumption and $t f(t) \rightarrow 0$ as $t \rightarrow \infty$ since $\lim _{t \rightarrow \infty} t f(t)$ and $\lim _{t \rightarrow \infty} F(t)$ exist. Also, by L'Hospital's rule

$$
\lim _{t \rightarrow \infty} \frac{f(t)}{[t f(t)]^{\prime}} \cdot \frac{t f(t)}{E(t)}=1
$$

Therefore

$$
\lim _{t \rightarrow \infty} \frac{t f^{2}(t)}{\left[t f^{\prime}(t)+p f(t)\right] E(t)}=\frac{u}{u+p-1}
$$

where

$$
u=\lim _{t \rightarrow \infty} \frac{[t f(t)]^{\prime}}{f(t)} .
$$

Hence, if $p=1, C_{p}[F(t)]$ converges more rapidly than $F(t)$ to $F(\infty)$ by Theorem 6 . Now to show the remaining part of the theorem we note that $p-1 \leqq 0$ and hence if $u<0,(2.25)$ satisfies Theorem 5 and the result is established. Since $\lim _{t \rightarrow \infty}$ $f(t) /[t f(t)]^{\prime}$ exists, $u \neq 0$. Now suppose $u>0$. Then there exists a $T$ such that $[t f(t)]^{\prime} / f(t)>0$ when $t>T$.

Simplifying (2.26) we have

$$
f^{\prime}(t) / f(t)>-1 / t, \quad t>T .
$$

Now if $f(t) \neq 0$ for $t>T$ we have for $t_{1}>t_{2}>T$

$$
\ln \left|\frac{f\left(t_{1}\right)}{f\left(t_{2}\right)}\right|>\ln \frac{t_{2}}{t_{1}}
$$

or

$$
t_{1}\left|f\left(t_{1}\right)\right|>t_{2}\left|f\left(t_{2}\right)\right|>0 .
$$

However, since $f(t)$ has only a countable number of zeros, we can select $t_{1}>t_{2}>T$ so that $f(t) \neq 0, t \in\left[t_{2}, t_{1}\right]$ and $(2.29)$ still holds. But the latter implies $\lim _{t \rightarrow \infty}$ $t f(t) \neq 0$ which cannot be since $F(t)$ converges as $t \rightarrow \infty$ and $\lim _{t \rightarrow \infty} t f(t)$ exists. Hence $u<0$ and

$$
0<u /(u+p-1) \leqq 1
$$


and the theorem follows. Example 1 below shows that if $p \neq 1, C_{p}[F(t)]$ may not converge more rapidly to $F(\infty)$ than $F(t)$ even though the conditions of Theorem 8 are met. However, we do have the following generalization of the second part of Theorem 8.

ThEOREM 9. If $\lim _{t \rightarrow \infty}\left[t^{p} f(t)\right]$ exists and $\lim _{t \rightarrow \infty} f(t) /\left[t^{p} f(t)\right]^{\prime}$ exists and is different from zero, then $C_{p}[F(t)]$ converges more rapidly than $F(t)$ to $F(\infty)$.

Proof. The proof follows much the same line as the case $p=1$ in Theorem 7 .

A natural question which arises in regard to transformations such as $C_{p}[F(t)]$ is the question of exactness. That is, for what functions is $C_{p}[F(t)] \equiv F(\infty)$ for some $p$ and all $t>t_{0} \geqq a$. In regard to this we have the following theorem.

Theorem 10. A necessary and sufficient condition that there exists a $t_{0}$ such that

$$
C_{p}[F(t)] \equiv F(\infty), \quad \text { if } t>t_{0}
$$

is that

$$
f(t)=c_{1} t^{-p} e^{-c_{2} t^{1-p}}, \quad p \neq 1,
$$

and

$$
f(t)=c_{3} t^{-c_{4}}, \quad p=1,
$$

when $t>t_{0}$. The quantities $c_{1}, c_{2}, c_{3}$ and $c_{4}$ are arbitrary constants satisfying the conditions $c_{2}>0$ and $c_{4}>1$.

Proof. Suppose there exists a $t_{0}$ such that (2.31) holds. Then

$$
F(t)-F(\infty)=t f^{2}(t) /\left(t f^{\prime}(t)+p f(t)\right), \quad t>t_{0} .
$$

Now letting $y=F(t)-F(\infty)$ we can write (2.34) in the form

$$
y=t\left(y^{\prime}\right)^{2} /\left(t y^{\prime \prime}+p y^{\prime}\right), \quad t>t_{0} .
$$

Solving this equation yields (2.32) and (2.33) as necessary conditions for the appropriate value of $p$. Substitution in (2.31) easily shows the sufficiency.

Example 1. Let

$$
F(t)=\int_{a}^{t} x^{-\alpha} d x, \quad \alpha>1
$$

By Theorem $10 C_{1}$ is exact and $C_{1}[F(t)] \equiv\left(a^{1-\alpha}\right) /(1-\alpha)$, for all $t>a>0$. It should be noted that for this function if $p \neq 1$ then $C_{p}$ is not exact. In fact, if $p \neq 1$, $C_{p}[F(t)]$ does not converge more rapidly than $F(t)$, although it does converge uniformly better than $F(t)$ to $F(\infty)$. Another comment worth making is that in this case it can easily be shown that the error in approximating $F(\infty)$ by $C_{p}[F(t)]$ is a monotone decreasing function of $p$.

Example 2.

$$
F(t)=\frac{2}{\sqrt{ } \pi} \int_{a}^{t} e^{-x^{2}} d x \rightarrow \operatorname{erfc}(a) \quad \text { as } t \rightarrow \infty
$$

where $0<a<\infty$.

In this case 


$$
\lim _{t \rightarrow \infty} \frac{f(t)}{f^{\prime}(t)}=0
$$

so that Theorem 7 is of no value. However, it is easy to show (without knowing a closed form for $E(t))$ that

$$
\lim _{t \rightarrow \infty} \frac{t f^{2}(t)}{\left[t f^{\prime}(t)+p f(t)\right] E(t)}=\lim _{t \rightarrow \infty} \frac{e^{-t^{2}}}{(2 t) \int_{t}^{\infty} e^{-x^{2}} d x}=1,
$$

so that by Theorem $6 C_{p}[F(t)]$ converges more rapidly than $F(t)$ to erfc $(a)$.

In particular suppose $a=1$ and $p=0$. Then

$$
F(t)=\frac{2}{\sqrt{ } \pi} \int_{1}^{t} e^{-x^{2}} d x
$$

and

$$
C_{0}[F(t)]=\frac{2}{\sqrt{ } \pi} \int_{1}^{t} e^{-x^{2}} d x+\frac{1}{\sqrt{ } \pi} \frac{e^{-t^{2}}}{t} .
$$

Thus, for example, we have the following:

\begin{tabular}{ccccc}
\multicolumn{5}{c}{$F(\infty) \cong .15729921$} \\
$t$ & $F(t)$ & Error & $C_{0}[F(t)]$ & Error \\
\hline 1.0 & 0.0 & .15729921 & .20755375 & .05025454 \\
1.5 & .11340435 & .04389487 & .15304778 & .00425144 \\
2.0 & .15262147 & .00467775 & .15778822 & .00048900
\end{tabular}

III. Iterated $C_{p}$ Transformations. Thus far we have only considered $C_{p}$ applied to $F$ one time. It is interesting to consider the possibility of applying $C_{p}$ repeatedly to $F$. Of course, this is not directly possible since $C_{p}[F(t)]$ is not in the domain of $C_{p}$. However, by noting that

$$
C_{p}[F(t)]=\int_{a}^{t}\left\{f(x)-\left[\frac{x f^{2}(x)}{x f^{\prime}(x)+p f(x)}\right]^{\prime}\right\} d x-\frac{a f^{2}(a)}{a f^{\prime}(a)+p f(a)}
$$

for functions with appropriate differentiability properties, we see that it would be possible to define what we might consider an iterated $C_{p}$ transformation. Thus it would seem logical to define $C_{p}{ }^{2}$ by

$$
C_{p}^{2}[F(t)]=C_{p} \int_{a}^{t}\left\{f(x)-\left[\frac{x f^{2}(x)}{x f^{\prime}(x)+p f(x)}\right]^{\prime}\right\} d x-\frac{a f^{2}(a)}{a f^{\prime}(a)+p f(a)}
$$

This leads us to the following definition.

Definition 4 . Let $A$ and $B$ be operators defined by

$$
A \phi(t)=\frac{t^{p} \phi^{2}(t)}{\left[t^{p} \phi(t)\right]^{\prime}}
$$

and 


$$
B \phi(t)=\phi(t)-[A \phi(t)]^{\prime}
$$

Then we define $C_{p}{ }^{n}$ by

$$
C_{p}^{n}[F(t)]=F(t)-\sum_{k=0}^{n-1} A B^{k} f(t),
$$

provided $A B^{k} f(t)$ is defined and finite for every $t \in[a, \infty), k=0, \cdots, n-1$. From the above it is clear that $C_{p}{ }^{n}[F(t)] \rightarrow F(\infty)$ as $t \rightarrow \infty$ if and only if

$$
\lim _{t \rightarrow \infty} \sum_{k=0}^{n-1} A B^{k} f(t)=0
$$

Now notice that

$$
\int_{a}^{t} B^{n}[f(x)] d x=F(t)+\sum_{k=0}^{n-1} A\left\{B^{k}[f(a)]\right\}-\sum_{k=0}^{n-1} A\left\{B^{k}[f(t)]\right\} .
$$

Hence we could write

$$
C_{p}^{n}[F(t)]=\int_{a}^{t} B^{n}[f(x)] d x-\sum_{k=0}^{n-1} A\left\{B^{k}[f(a)]\right\}
$$

and thus for functions such that

$$
\left.\lim _{n \rightarrow \infty} \int_{a}^{\infty} B_{\llcorner\mathcal{L}}^{n}(t)\right] d t=0,
$$

and (3.6) holds for every $k$, we have

$$
F(\infty)=\int_{a}^{\infty} f(t) d t=-\sum_{k=0}^{\infty} A\left\{B^{k}[f(a)]\right\} .
$$

In general it may be difficult to determine just when (3.10) is valid. However, this does not mean it cannot be used to suggest approximating functions for $F(\infty)$ that may be checked for validity in other ways. This is illustrated in the examples which follow.

Example 3. Consider the right tail of the Chi-square distribution function, i.e., $Q\left(\chi^{2} \mid \nu\right)$ where

$$
Q\left(\chi^{2} \mid \nu\right)=Q(2 u \mid 2 v)=\frac{1}{\Gamma(v)} \int_{u}^{\infty} e^{-x} x^{v-1} d x .
$$

The two-term approximating sum with $p=0$ (i.e. applying $C_{0}^{2}$ ) is

$$
Q(2 u \mid 2 v) \cong C(2 u, 2 v)=\frac{e^{-(u-v)}}{2 \pi b}\left(\frac{u}{v}\right)^{v}\left[1-\frac{v-1}{b^{2}+2 u}\right]-\frac{12 v^{3 / 2}}{12 v+1},
$$

where $b=u-v+1$. This approximation has been studied by Gray, Thompson and McWilliams [12]. They found it to be quite accurate over most of the range of values of $\chi^{2}$ and $\nu$ for which $Q\left(\chi^{2} \mid \nu\right) \leqq .1$. A sample of the results of this approximation are given in the following table. The value of $C\left(\chi^{2}, \nu\right)$ was computed for $\nu=1,2,5,10,20,30,100,250,500$, using the values of $\chi^{2}$ which yielded $Q\left(\chi^{2} \mid \nu\right)=$ $.1, .05$ and .01 . 


\begin{tabular}{rlr}
\multicolumn{1}{r}{$\nu$} & $Q$ & $C\left(\chi^{2}, \nu\right)-Q$ \\
\hline 1 & .1 & -.00105 \\
1 & .05 & -.00028 \\
1 & .01 & -.00002 \\
2 & .1 & .00010 \\
2 & .05 & .00005 \\
2 & .01 & .00001 \\
5 & .1 & .00054 \\
5 & .05 & .00013 \\
5 & .01 & .00000 \\
10 & .1 & .00058 \\
10 & .05 & .00010 \\
10 & .01 & .00000 \\
30 & .1 & .00038 \\
30 & .05 & .00004 \\
30 & .01 & -.00001 \\
100 & .1 & .00007 \\
100 & .05 & -.00022 \\
100 & .01 & -.00004 \\
250 & .1 & -.00001 \\
250 & .05 & -.00033 \\
250 & .01 & -.00006 \\
500 & .1 & -.00018 \\
500 & .05 & -.00043 \\
500 & .01 & -.00007
\end{tabular}

For another example of the iterated $C_{0}$ see [10].

Example 4.

$$
F(\phi, k)=\int_{0}^{\phi}\left(1-k^{2} \sin ^{2} t\right)^{-1 / 2} d t
$$

and we define

$$
\begin{aligned}
& m=k^{2} \\
& a=\left[\frac{(2-m)}{1+m}\right]^{1 / 3}>0, b=\left[\frac{(1-2 m)}{(m-2)(m+1)}\right]^{1 / 3}, \\
& C=\left|\frac{(1+m)^{2}}{m-2}\right|^{1 / 3}<0, x=C+\frac{a-C}{\sin ^{2} \phi}, \\
& h=a\left[C+\frac{b(2 m-1)}{m-2}\right]<0, g=2 m-1, \\
& S=2\left[\frac{2-m}{3 a}\right]^{1 / 2}, r(x)=x^{3}+h x+g,
\end{aligned}
$$

and 


$$
I(x)=\int_{x}^{\infty}[r(t)]^{-1 / 2} d t
$$

then (see [7])

$$
F(\phi, k)=S^{-1} I(x) .
$$

Now using $p=1$, which is certainly suggested by Theorem 8 if $n=1$, and taking only the first term in (3.10) as an approximation for $I(x)$ we have

$$
\hat{I}(x)=2 x C_{2}^{1 / 2}(x) / C_{3}(x),
$$

where

$$
\begin{aligned}
& C_{2}(x)=x^{3}+h x+g \\
& C_{3}(x)=x^{3}-h x-2 g .
\end{aligned}
$$

The approximation (3.14) is undoubtedly quite rough. However, the following table, with $k=\sin \alpha$, indicates $\hat{I}(x)$ is better than one might expect.

\begin{tabular}{rrrr}
$\alpha$ & $\phi$ & $F(\phi, \alpha)$ & $\hat{F}(\phi, \alpha)$ \\
\hline $2^{\circ}$ & $5^{\circ}$ & .087264 & .087271 \\
$2^{\circ}$ & $10^{\circ}$ & .174534 & .174651 \\
$2^{\circ}$ & $20^{\circ}$ & .349074 & .351937 \\
$4^{\circ}$ & $5^{\circ}$ & .087267 & .087271 \\
$4^{\circ}$ & $10^{\circ}$ & .174537 & .174654 \\
$4^{\circ}$ & $10^{\circ}$ & .349100 & .351952 \\
$10^{\circ}$ & $5^{\circ}$ & .087270 & .087274 \\
$10^{\circ}$ & $10^{\circ}$ & .174559 & .174673 \\
$10^{\circ}$ & $20^{\circ}$ & .349275 & .352058 \\
$30^{\circ}$ & $5^{\circ}$ & .087294 & .087297 \\
$30^{\circ}$ & $10^{\circ}$ & .174754 & .174850 \\
$30^{\circ}$ & $20^{\circ}$ & .350819 & .353172
\end{tabular}

Department of Mathematics

Texas Technology College

Lubbock, Texas 74904

Department of Statistics

Southern Methodist University

Dallas, Texas 75222

1. S. Lubkin, "A method of summing infinite series," J. Res. Nat. Bur. Standards, v. 48, 1952 , pp. 228-254. MR 14, 500.

2. D. Shanks, "Non-linear transformations of divergent and slowly convergent sequences," J. Math. Phys., v. 34, 1955, pp. 1-42. MR 16, 961 .

3. P. WYNN, "On a device for computing the $e_{m}\left(S_{n}\right)$ transformation," $M T A C$, v. 10, 1956, pp. 91-96. MR 18, 801 .

4. P. WYNN, "The epsilon algorithm and operational formulas of numerical analysis," Math. Comp., v. 15, 1961, pp. 151-158. MR 28 \#1736.

5. P. WYNN, "On the convergence and stability of the epsilon algorithm," SIAM J. Numer. Anal., v. 3, 1966, no. 1, pp. 91-122. MR $34 \# 6996$.

6. H. L. GRAY \& T. A. A TCHIson, "Nonlinear transformations related to the evaluation of improper integrals. I," SIAM J. Numer. Anal., v. 4, 1967, pp. 363-371. MR 36 \#6145. 
7. W. G. FAIR \& Y. L. LUKE, "Rational approximations to the incomplete elliptic integrals of the first and second kinds," Math. Comp., v. 21, 1967, pp. 418-422. MR 36 \#5400.

8. T. A. ATCHISON \& H. L. GRAY, "Nonlinear transformations related to the evaluation of improper integrals. II," SIAM J. Numer. Anal., v. 5, 1968, pp. 451-459. MR 37 \#4941.

9. W. R. SchuCANY \& H. L. GRAY, "A new approximation related to the error function," Math. Comp., v. 22, 1968, pp. 201-202.

10. H. L. Gray \& W. R. Schucany, "On the evaluation of distribution functions," J. Amer. Statist Assoc., v. 63, 1968, pp. 715-720.

11. H. L. Gray \& T. A. Atchison, "The generalized G-transform," Math. Comp., v. 22, 1968, pp. 595-605. MR 37 \#4942.

12. H. L. Gray, R. W. Thompson \& G. V. McWilliams, "A new approximation for the chi-square integral," Math. Comp., v. 23, 1969, pp. 85-89. 